reminder that it can be difficult to interpret intention to treat analyses when large proportions of patients did not complete their assigned protocol.

Overall, the book is a superb guide to reporting statistical results in the medical literature. It has exceptional additional value regarding critically designing studies and appraising the medical literature. The breadth of its audience is large and includes research fellows ("every fellow should have a copy"), investigators at most levels, and all faculty, including clinical educators, to use in their interpretation of the literature and clinical teaching. The second edition has a wonderful mix of text, tables, and figures to promote learning and retention. The writing is efficient and flows remarkably easily. The statistics are focused on concepts presented in language, tables, and figures that are easy to understand and teach succinctly. Best of all, the book is easy to pick up and put down, and to read short sections productively as needs determine and time allows.

JOHN E CORNELL, PHD VALERIE A LAWRENCE, MD, MSC South Texas Veterans Health Care System and University of Texas Health Science Center at San Antonio San Antonio, Texas, USA

\title{
Resource review
}

Evans I, Thornton H, Chalmers I. Testing treatments. better research for better healthcare. London: The British Library, 2006. ISBN $071234909 \mathrm{X}$.

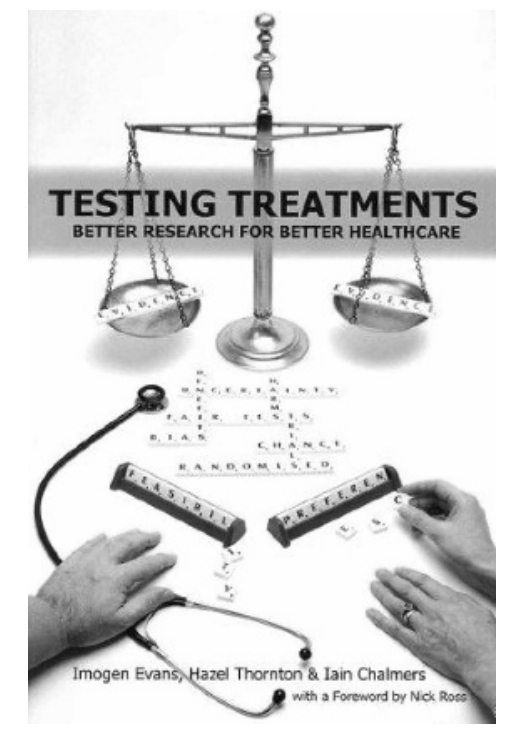

Testing treatments: better research for better healthcare can be obtained from the British Library Shop

(bl-bookshop@bl.uk) for £12.95.
A medical journalist, a critical patient, and a well known scientist summarise impressive examples in their book entitled Testing treatments: better research for better healthcare.

This book is written for non-scientists. It is a must for all who want to understand and critically appraise health care or want to become adequate partners for their doctors in shared decision making. For practising doctors, it is an ideal refresher of their previous university courses. The quality of information is simply the best available.

The first chapter describes the results of new, but not necessarily better, treatments, such as blindness in prematurely born babies associated with inappropriate use of oxygen therapy, deaths caused by placing babies prone to sleep, or the appropriate description of advantages and risks associated with hormone therapy in menopausal women.

The second chapter discusses inadequately tested therapies, such as radical mastectomy and high dose chemotherapy. We should learn from these lessons that a treatment has to be tested more extensively the more harm is associated with its expected benefit.

The key concepts for testing treatments are explained in the third and fourth chapters. The language used in these chapters is readily comprehensible. It is, therefore, surprising to learn that some researchers handle the problem of uncertainty rather cavalierly-for exanple, by continuing to recommend antiarrhythmic treatment despite existing evidence to the contrary. This chapter impressively demonstrates the systematic mistakes (bias) that will continue to be made unless the writer and reader of scientific articles are aware of these risks.

Not only doctors but also patients should be able to understand the difference between good, bad, and unnecessary research as outlined in chapters 5 and 6 . The development of modern medicine requires that patients participate in decision making. Firstly, however, they must understand the recommendations of the other stakeholders in the system, such as doctors who rest assured in the correctness of their specialty, industry which is committed to its products, and hospital managers who are convinced of the necessity to employ technical advancements. Only informed patients can contribute to improvement in the testing of treatments (chapter 7).

The blueprint for a revolution (chapter 8) is nothing other than the hint that the informed patient is the best guarantee for high quality and safe medical care. References complete the 115 pages of text, which I found important enough to look for a German translator, publisher, and sponsor to make this valuable information available to the non-English-speaking German readership. 